\title{
34. MATHEMATICAL TREATMENT OF GEOCHEMICAL DATA, DEEP SEA DRILLING PROJECT SITES 415 AND 416
}

\author{
P. Debrabant, J. Foulon, and H. Maillot, U.E.R. des Sciences de la Terre, \\ Université des Sciences et Techniques de Lille, 59650 Villeneuve d'Ascq, France
}

\begin{abstract}
We collected geochemical data from 94 samples from DSDP Leg 50 . With the help of multivariate analyses (correlations, factor analysis in principal components) we stress the essential characteristics of the geochemical environment of Sites 415 and 416, and show analogies with the other DSDP holes of the eastern Atlantic margin. Sediments from Site 416 are dominantly ferro-potassic, a detrital deposit containing primary Cretaceous minerals. The Site 415 sediments are more ferro-magnesian; there is a correspondence with prevalent smectites marked by the same trace elements ( $\mathrm{Ti} \mathrm{Cr} \mathrm{V}$ ) at Sites 398 to 402 . Contents of the major elements are similar, showing continuity of the origin of the detrital supply.

The environment became more open between Albian and Aptian time. Sediments of the two sites show numerous similarities from that level upward and a geochemical break is indicated somewhere between Paleocene and Miocene.
\end{abstract}

\section{MATHEMATICAL TREATMENT OF THE GEOCHEMICAL DATA}

\section{Introduction}

The predominance of detrital material in the sediments analyzed makes a precise geochemical study and establishment of a detailed geochemical zonation difficult. Our analyses were made from the same samples as those analyzed by Chamley (this volume) and we shall refer to the mineralogical results he obtained.

The results of the geochemical analyses are shown in Tables 1 and 3.

\section{General Considerations on the Geochemistry of Leg $\mathbf{5 0}$}

Site 415 (Tables 1 and 2)

The very sparse sampling does not permit great precision in the study of samples from this site. Most of the samples fall into a relatively dense cluster in the $\mathrm{Al}_{2} \mathrm{O}_{3}$, $\mathrm{Fe}_{2} \mathrm{O}_{3}, \mathrm{MgO}$ diagram (Figure 1); a few more-magnesian or more-ferruginous samples are isolated. The data points of lithologic Units IV, V, VI are more markedly dispersed. The study shows that the corresponding samples from Units I, II, and III are geochemically different and show a marked discontinuity between Paleocene and Miocene sediments.

The Miocene and overlying sediments, as seen from the $\mathrm{Sr} / \mathrm{CaO}$ ratio, are usually calcareous $(50$ to $70 \%$ $\mathrm{CaCO}_{3}$ ) accompanied by high strontium concentrations. The calcareous samples of the lower series do not have high strontium concentrations. This is a general tendency in the sediment of the eastern Atlantic margin sites (Chamley et al., 1979; Debrabant et al., 1979).
In addition, certain transition elements $(\mathrm{Mn}, \mathrm{Cr}, \mathrm{V})$ occur in lower concentrations or in different associations within the Paleocene and Miocene sediments. In Miocene and overlying sediments, the occurrence of manganese does not seem to be related to that of carbonates. This can be caused either by a difference in the degree of carbonate diagenesis or to a distinct change in the redox conditions of the depositional environment ( $a$ more reducing environment in the lower series).

Furthermore, the $\mathrm{MgO} / \mathrm{K}_{2} \mathrm{O}, \mathrm{Al}_{2} \mathrm{O}_{3} / \mathrm{K}_{2} \mathrm{O}$, and $\mathrm{Fe}_{2} \mathrm{O}_{3} /$ $\mathrm{K}_{2} \mathrm{O}$ ratios decrease toward the top of the hole owing to an increase in the supply of potassic clay and a relative decrease in smectites. (The illite increases more rapidly than the chlorite, which masks the ferromagnesian composition of that clay.) Lastly, the transition from Cretaceous to Tertiary sediments is accompanied by a slight increase of phosphate occurring in the Cenomanian (only traces in Sample 415A-8-1, $110 \mathrm{~cm}$ ), reaching 0.57 per cent $\mathrm{P}_{2} \mathrm{O}_{5}$ in the Paleocene and diminishing in the Miocene $(0.23 \%$ in Sample $415-5-3,40 \mathrm{~cm})$.

A similar phenomenon occurs in sediments of approximately the same age from Site 416 , and $\mathrm{P}_{2} \mathrm{O}_{5}$ is also traceable in the Cenomanian and Paleocene at Site 398 (Debrabant et al., unpublished manuscript). This might have resulted from a pre-existing deep current or the initiation of a new current, as is suggested by the presence of siliceous organisms in the sediments of both sites.

\section{Site 416 (Tables 3, 4)}

All samples analyzed contained very low concentrations of carbonate. The greatest amounts of 63 per cent and 73 per cent occur in upper Tithonian and Miocene 
TABLE 1

Geochemical Analyses, Site $415^{\mathrm{a}}$

\begin{tabular}{|c|c|c|c|c|c|c|c|c|c|c|c|c|c|c|c|c|c|c|c|}
\hline $\begin{array}{c}\text { Sample } \\
\text { (interval in } \mathrm{cm} \text { ) }\end{array}$ & $\mathrm{SiO}_{2}$ & $\mathrm{Al}_{2} \mathrm{O}_{3}$ & $\mathrm{CaO}$ & $\mathrm{MgO}$ & $\begin{array}{c}\mathrm{Na}_{2} \mathrm{O} \\
(\%)\end{array}$ & $\mathrm{K}_{2} \mathrm{O}$ & $\mathrm{TiO}_{2}$ & $\mathrm{P}_{2} \mathrm{O}_{5}$ & $\mathrm{Fe}_{2} \mathrm{O}_{3}$ & $\mathrm{Mn}$ & $\mathrm{Zn}$ & $\mathrm{Li}$ & $\mathrm{Ni}$ & $\underset{(\mathrm{ppm})}{\mathrm{Cr}}$ & $\mathrm{Sr}$ & Co & $\mathrm{Cu}$ & $\mathrm{Pb}$ & V \\
\hline $\begin{array}{l}415 A-1-5,100 \\
415 A-1-6,14\end{array}$ & $\begin{array}{l}18.00 \\
24.30\end{array}$ & $\begin{array}{l}6.26 \\
7.85\end{array}$ & $\begin{array}{l}34.90 \\
29.19\end{array}$ & $\begin{array}{l}1.78 \\
2.13\end{array}$ & $\begin{array}{l}1.55 \\
1.67\end{array}$ & $\begin{array}{l}1.54 \\
1.91\end{array}$ & $\begin{array}{l}0.31 \\
0.40\end{array}$ & & $\begin{array}{l}2.52 \\
3.33\end{array}$ & $\begin{array}{l}260 \\
260\end{array}$ & $\begin{array}{l}74 \\
76\end{array}$ & $\begin{array}{l}37 \\
45\end{array}$ & $\begin{array}{l}35 \\
31\end{array}$ & $\begin{array}{l}39 \\
49\end{array}$ & $\begin{array}{r}1150 \\
900\end{array}$ & $\begin{array}{l}16 \\
14\end{array}$ & $\begin{array}{l}64 \\
17\end{array}$ & $\begin{array}{l}37 \\
18\end{array}$ & $\begin{array}{r}79 \\
105\end{array}$ \\
\hline $\begin{array}{l}415 B-2-1,44 \\
415 B-2-1,91 \\
415 B-3-1,62 \\
415 B-3-3,62 \\
415 B-4-1,6 \\
415 B-5-3,40 \\
415 B-5-5,33 \\
415 B-5-6,27\end{array}$ & $\begin{array}{l}12.15 \\
20.15 \\
16.95 \\
24.55 \\
17.50 \\
23.50 \\
30.35 \\
23.00\end{array}$ & $\begin{array}{l}3.84 \\
6.31 \\
4.72 \\
6.32 \\
4.96 \\
4.25 \\
6.61 \\
4.13\end{array}$ & $\begin{array}{l}40.60 \\
33.08 \\
36.19 \\
30.45 \\
35.77 \\
32.90 \\
25.94 \\
33.60\end{array}$ & $\begin{array}{l}1.33 \\
2.04 \\
1.73 \\
1.78 \\
1.90 \\
1.58 \\
2.48 \\
1.60\end{array}$ & $\begin{array}{l}1.11 \\
1.26 \\
1.13 \\
1.18 \\
1.01 \\
0.98 \\
1.20 \\
1.11\end{array}$ & $\begin{array}{l}1.14 \\
1.63 \\
1.33 \\
1.55 \\
1.45 \\
1.16 \\
1.69 \\
1.10\end{array}$ & $\begin{array}{l}0.20 \\
0.32 \\
0.24 \\
0.33 \\
0.23 \\
0.22 \\
0.36 \\
0.21\end{array}$ & $\begin{array}{l}\text { n.d. } \\
\text { n.d. } \\
\text { n.d. } \\
\text { n.d. } \\
\text { n.d. } \\
0.23 \\
\text { n.d. } \\
\text { n.d. }\end{array}$ & $\begin{array}{l}1.64 \\
2.50 \\
2.06 \\
2.50 \\
2.13 \\
1.33 \\
3.14 \\
1.69\end{array}$ & $\begin{array}{l}290 \\
210 \\
200 \\
150 \\
200 \\
250 \\
200 \\
150\end{array}$ & $\begin{array}{l}33 \\
79 \\
50 \\
64 \\
53 \\
69 \\
79 \\
55\end{array}$ & $\begin{array}{l}23 \\
42 \\
34 \\
37 \\
31 \\
34 \\
53 \\
36\end{array}$ & $\begin{array}{l}23 \\
36 \\
35 \\
35 \\
35 \\
34 \\
35 \\
25\end{array}$ & $\begin{array}{l}37 \\
43 \\
41 \\
45 \\
44 \\
39 \\
58 \\
45\end{array}$ & $\begin{array}{r}1350 \\
1200 \\
1350 \\
1250 \\
1350 \\
1050 \\
850 \\
1200\end{array}$ & $\begin{array}{r}8 \\
15 \\
15 \\
13 \\
13 \\
13 \\
9 \\
9\end{array}$ & $\begin{array}{l}12 \\
13 \\
18 \\
16 \\
18 \\
22 \\
21 \\
16\end{array}$ & $\begin{array}{r}24 \\
21 \\
21 \\
6 \\
32 \\
25 \\
22 \\
24\end{array}$ & $\begin{array}{r}47 \\
89 \\
84 \\
105 \\
47 \\
37 \\
47 \\
58\end{array}$ \\
\hline $\begin{array}{l}415 A-5, C C, 1 \\
415 A-6-1,112 \\
415 A-7-1,24 \\
415 A-8-1,110 \\
415 A-9-1,95 \\
415 A-10-1,78 \\
415 A-11-1,12 \\
415 A-12-1,113 \\
415 A-13-1,125\end{array}$ & $\begin{array}{l}63.20 \\
32.90 \\
57.70 \\
44.35 \\
31.90 \\
45.50 \\
38.20 \\
42.35 \\
21.35\end{array}$ & $\begin{array}{r}13.60 \\
3.48 \\
15.35 \\
13.60 \\
10.27 \\
13.93 \\
12.81 \\
12.40 \\
5.37\end{array}$ & $\begin{array}{r}0.88 \\
27.86 \\
1.86 \\
9.66 \\
22.05 \\
8.51 \\
15.86 \\
13.86 \\
34.82\end{array}$ & $\begin{array}{l}2.82 \\
1.60 \\
3.17 \\
3.92 \\
1.80 \\
3.87 \\
2.30 \\
1.86 \\
1.61\end{array}$ & $\begin{array}{l}2.24 \\
1.42 \\
1.95 \\
1.60 \\
1.15 \\
1.16 \\
1.21 \\
1.40 \\
0.59\end{array}$ & $\begin{array}{l}1.44 \\
0.56 \\
2.14 \\
1.20 \\
0.66 \\
2.35 \\
0.90 \\
0.96 \\
0.65\end{array}$ & $\begin{array}{l}0.56 \\
0.13 \\
0.67 \\
0.75 \\
0.70 \\
0.70 \\
0.79 \\
0.83 \\
0.33\end{array}$ & $\begin{array}{l}0.23 \\
0.57 \\
0.43 \\
\text { n.d. } \\
\text { n.d. } \\
\text { n.d. } \\
\text { n.d. } \\
\text { n.d. } \\
\text { n.d. }\end{array}$ & $\begin{array}{l}4.64 \\
1.39 \\
6.07 \\
5.64 \\
4.50 \\
6.26 \\
6.00 \\
5.36 \\
6.14\end{array}$ & $\begin{array}{l}150 \\
650 \\
300 \\
600 \\
380 \\
530 \\
400 \\
300 \\
650\end{array}$ & $\begin{array}{r}150 \\
67 \\
130 \\
69 \\
66 \\
89 \\
84 \\
89 \\
47\end{array}$ & $\begin{array}{l}36 \\
25 \\
42 \\
30 \\
20 \\
46 \\
40 \\
34 \\
20\end{array}$ & $\begin{array}{l}48 \\
31 \\
58 \\
42 \\
27 \\
35 \\
25 \\
31 \\
18\end{array}$ & $\begin{array}{r}200 \\
95 \\
150 \\
120 \\
83 \\
110 \\
90 \\
90 \\
100 \\
47\end{array}$ & $\begin{array}{l}350 \\
750 \\
350 \\
400 \\
550 \\
350 \\
450 \\
600 \\
650\end{array}$ & $\begin{array}{r}17 \\
2 \\
18 \\
13 \\
16 \\
18 \\
15 \\
15 \\
16\end{array}$ & $\begin{array}{r}250 \\
33 \\
61 \\
4 \\
1 \\
16 \\
1 \\
1 \\
1\end{array}$ & $\begin{array}{l}14 \\
13 \\
16 \\
16 \\
30 \\
89 \\
13 \\
12 \\
25\end{array}$ & $\begin{array}{r}175 \\
63 \\
180 \\
160 \\
120 \\
130 \\
170 \\
140 \\
89\end{array}$ \\
\hline
\end{tabular}

aOrganic carbon not determined.

TABLE 2

Geochemical Ratios, Site 415

\begin{tabular}{|c|c|c|c|c|c|c|c|c|}
\hline $\begin{array}{c}\text { Sample } \\
\text { (interval in } \mathrm{cm} \text { ) }\end{array}$ & $\frac{\mathrm{SiO}_{2}}{\mathrm{Al}_{2} \mathrm{O}_{3}}$ & $\frac{\mathrm{Al}_{2} \mathrm{O}_{3}}{\mathrm{Fe}_{2} \mathrm{O}_{3}}$ & $\frac{\mathrm{MgO}}{\mathrm{K}_{2} \mathrm{O}}$ & $\frac{\mathrm{Al}_{2} \mathrm{O}_{3}}{\mathrm{~K}_{2} \mathrm{O}}$ & $\frac{\mathrm{Na}_{2} \mathrm{O}}{\mathrm{K}_{2} \mathrm{O}}$ & $\frac{\mathrm{Fe}_{2} \mathrm{O}_{3}}{\mathrm{~K}_{2} \mathrm{O}}$ & $\frac{\mathrm{Fe}_{2} \mathrm{O}_{3}}{\mathrm{MgO}}$ & $\frac{\mathrm{Sr} 10^{3}}{\mathrm{CaO}}$ \\
\hline $\begin{array}{l}415-1-5,100 \\
415-1-6,140\end{array}$ & $\begin{array}{l}2.9 \\
3.1\end{array}$ & $\begin{array}{l}2.5 \\
2.4\end{array}$ & $\begin{array}{l}1.2 \\
1.1\end{array}$ & $\begin{array}{l}4.1 \\
4.1\end{array}$ & $\begin{array}{l}1.01 \\
0.87\end{array}$ & $\begin{array}{l}1.6 \\
1.7\end{array}$ & $\begin{array}{l}1.4 \\
1.6\end{array}$ & $\begin{array}{l}3.3 \\
3.1\end{array}$ \\
\hline $\begin{array}{l}415 B-2-1,44 \\
415 B-2-1,91 \\
415 B-3-1,62 \\
415 B-3-3,62 \\
415 B-4-1,6 \\
415 B-5-3,4 \\
415 B-5-5,33 \\
415 B-5-6,27\end{array}$ & $\begin{array}{l}3.2 \\
3.2 \\
3.6 \\
3.9 \\
3.5 \\
5.5 \\
4.6 \\
5.6\end{array}$ & $\begin{array}{l}2.3 \\
2.5 \\
2.3 \\
2.5 \\
2.3 \\
3.2 \\
2.1 \\
2.4\end{array}$ & $\begin{array}{l}1.2 \\
1.3 \\
1.3 \\
1.2 \\
1.3 \\
1.4 \\
1.5 \\
1.5\end{array}$ & $\begin{array}{l}3.4 \\
3.9 \\
3.6 \\
4.1 \\
3.4 \\
3.7 \\
3.9 \\
3.8\end{array}$ & $\begin{array}{l}1.00 \\
0.77 \\
0.85 \\
0.76 \\
0.70 \\
0.84 \\
0.71 \\
1.01\end{array}$ & $\begin{array}{l}1.4 \\
1.6 \\
1.6 \\
1.6 \\
1.5 \\
1.2 \\
1.9 \\
1.5\end{array}$ & $\begin{array}{l}1.2 \\
1.2 \\
1.2 \\
1.4 \\
1.1 \\
0.8 \\
1.3 \\
1.1\end{array}$ & $\begin{array}{l}3.3 \\
3.6 \\
3.7 \\
4.1 \\
3.8 \\
3.2 \\
3.3 \\
3.6\end{array}$ \\
\hline $\begin{array}{l}415 \mathrm{~A}-5, \mathrm{CC}, 1 \\
415 \mathrm{~A}-6-1,112 \\
415 \mathrm{~A}-7-1,24 \\
415 \mathrm{~A}-8-1,110 \\
415 \mathrm{~A}-9-1,95 \\
415 \mathrm{~A}-10-1,78 \\
415 \mathrm{~A}-11-1,12 \\
415 \mathrm{~A}-12-1,113 \\
415 \mathrm{~A}-13-1,125\end{array}$ & $\begin{array}{l}4.7 \\
9.5 \\
3.8 \\
3.3 \\
3.1 \\
3.3 \\
3.0 \\
3.4 \\
4.0\end{array}$ & $\begin{array}{l}2.9 \\
2.5 \\
2.5 \\
2.4 \\
2.3 \\
2.2 \\
2.1 \\
2.3 \\
0.9\end{array}$ & $\begin{array}{l}2.0 \\
2.9 \\
1.5 \\
3.3 \\
2.7 \\
1.7 \\
2.6 \\
1.9 \\
2.5\end{array}$ & $\begin{array}{r}9.4 \\
6.2 \\
7.2 \\
11.3 \\
15.6 \\
5.9 \\
14.2 \\
12.9 \\
8.3\end{array}$ & $\begin{array}{l}1.56 \\
2.54 \\
0.91 \\
1.33 \\
1.74 \\
0.49 \\
1.34 \\
1.46 \\
0.91\end{array}$ & $\begin{array}{l}3.1 \\
2.5 \\
2.8 \\
4.7 \\
6.8 \\
2.7 \\
6.7 \\
5.6 \\
9.5\end{array}$ & $\begin{array}{l}2.1 \\
0.9 \\
1.9 \\
1.4 \\
2.5 \\
1.6 \\
2.6 \\
2.9 \\
3.8\end{array}$ & $\begin{array}{r}39.8 \\
2.7 \\
18.8 \\
4.4 \\
2.5 \\
4.1 \\
2.8 \\
4.3 \\
2.8\end{array}$ \\
\hline
\end{tabular}

samples, respectively. Conversely, many samples contain less than 5 per cent $\mathrm{CaCO}_{3}$. Complete dissolution could have occurred at two levels (Hauterivian and Aptian), but the samples do not have any resulting accumulations of metals.

The silicate phase, consequently, largely predominates in the cores, and high titanium concentrations confirm the detrital origin of most of the sediments. A few Tithonian to Valanginian samples and some Aptian to Barremian are particularly siliceous. Their high $\mathrm{SiO}_{2} / \mathrm{Al}_{2} \mathrm{O}_{3}$ ratios imply the presence of free silica.

Most of the samples from Units VI and VII are grouped in a very restricted area on the $\mathrm{Al}_{2} \mathrm{O}_{3}, \mathrm{Fe}_{2} \mathrm{O}_{3}$, $\mathrm{MgO}$ diagram (Deer et al., 1963). A few particularly ferriferous or magnesian samples appear as isolated points (Figure 2). On the other hand, most of the Eocene and
post-Eocene samples gather outside that zone. This tendency was also noted in the Site 415 samples.

A comparison between the respective domains of Sites 415 and 416 (Figure 2) also shows that the preAlbian samples clearly group toward the aluminum apex (Site 416), which implies distinct clay sources before and after that epoch. Kaolinite is important in this regard.

Samples from Site 416 do not contain significantly high concentrations of magnesium, which confirms the paucity of dolomite. Two slightly phosphatic levels occur in upper Eocene and Miocene sediments.

No zone is significantly enriched in iron or in transition metals. Lithium and vanadium are highly concentrated, however, compared to the sediments of Legs 47B and 48. Apparently this is related to a high illite content as it correlates with high values for $\mathrm{K}_{2} \mathrm{O}$. Finally, the organic carbon is traceable in quantities larger than 1 per cent only in the Hauterivian sediments.

\section{Mathematical Treatment of the Geochemical Data}

We shall not compare the data from the multivariate studies between sites because only the post-Aptian sequence is common to both Sites 415 and 416 . This comprises only eight samples, whereas we treat 75 samples in the mathematical study.

\section{Mean Chemical Compositions (Table 5)}

The standard deviations are naturally high because of the heterogeneity of the materials analyzed. However, sediments from Site 416 tend to have more silicate and less carbonate. A study of the probable variation range, however, shows that the chemical-element concentrations, except for potassium and lithium, all overlap. The particular relationship between those two elements is 
TABLE 3

Geochemical Analyses, Site 416

\begin{tabular}{|c|c|c|c|c|c|c|c|c|c|c|c|c|c|c|c|c|c|c|c|c|}
\hline $\begin{array}{c}\text { Sample } \\
\text { (interval in } \mathrm{cm} \text { ) }\end{array}$ & $\mathrm{SiO}_{2}$ & $\mathrm{Al}_{2} \mathrm{O}_{3}$ & $\mathrm{CaO}$ & $\mathrm{MgO}$ & $\mathrm{Na}_{2} \mathrm{O}$ & $\mathrm{K}_{2} \mathrm{O}$ & $\mathrm{TiO}_{2}$ & $\mathrm{P}_{2} \mathrm{O}_{5}$ & $\mathrm{Fe}_{2} \mathrm{O}_{3}$ & $\mathrm{C}_{\text {org }} \mathrm{a}^{\mathrm{a}}$ & $\mathrm{Mn}$ & $\mathrm{Zn}$ & $\mathrm{Li}$ & $\mathrm{Ni}$ & $\mathrm{Cr}$ & $\mathrm{Sr}$ & Co & $\mathrm{Cu}$ & $\mathrm{Pb}$ & V \\
\hline $416-1-1,33$ & 6.60 & 8.09 & 27.65 & 1.94 & 1.67 & 1.81 & 0.44 & n.d. & 3.50 & - & 370 & 77 & 41 & 29 & 54 & 900 & 15 & 34 & 110 & 105 \\
\hline $416-1-1,44$ & 13.10 & 3.89 & 40.60 & 1.36 & 0.89 & 1.23 & 0.14 & n.d. & 1.72 & 0.15 & 280 & 55 & 24 & 27 & 33 & 1380 & 14 & 15 & 320 & 32 \\
\hline $416-1-1,99$ & 38.25 & 7.49 & 17.36 & 4.71 & 1.30 & 2.45 & 0.31 & 0.23 & 3.70 & 0.14 & 400 & 110 & 52 & 28 & 76 & 450 & 9 & 28 & 22 & 63 \\
\hline $416-1-1,117$ & 32.75 & 8.44 & 21.98 & 2.82 & 1.48 & 2.21 & 0.29 & n.d. & 3.80 & - & 330 & 73 & 49 & 33 & 64 & 750 & 13 & 15 & 95 & 100 \\
\hline $416-1-2,33$ & 27.80 & 6.09 & 28.21 & 2.33 & 1.16 & 1.58 & 0.20 & n.d. & 2.63 & 0.18 & 390 & 71 & 47 & 26 & 48 & 1000 & 9 & 33 & 32 & 42 \\
\hline $416-2-1,31$ & 41.90 & 9.62 & 14.52 & 3.74 & 1.28 & 2.73 & 0.25 & n.d. & 4.45 & 0.25 & 350 & 83 & 72 & 20 & 77 & 480 & 6 & 16 & 9 & 74 \\
\hline $416-2-3,64$ & 40.65 & 8.44 & 17.50 & 2.88 & 1.36 & 2.45 & 0.26 & n.d. & 3.92 & 0.28 & 300 & 83 & 57 & 21 & 61 & 600 & 6 & 54 & 12 & 63 \\
\hline $416-3-3,39$ & 53.65 & 13.39 & 4.41 & 3.60 & 1.53 & 3.52 & 0.66 & n.d. & 5.22 & 0.22 & 320 & 107 & 79 & 31 & 125 & 280 & 13 & 29 & 25 & 120 \\
\hline $416-3-3,47$ & 41.90 & 9.38 & 12.60 & 3.65 & 1.28 & 2.85 & 0.60 & 0.34 & 4.43 & 0.71 & 340 & 95 & 59 & 45 & 120 & 440 & 15 & 15 & 22 & 110 \\
\hline $416-6-3,3$ & 65.65 & 14.31 & 0.21 & 1.83 & 1.45 & 3.60 & 0.71 & n.d. & 5.00 & 0.01 & 180 & 67 & 60 & 39 & 114 & 240 & 13 & 45 & 16 & 95 \\
\hline $416-7-2,122$ & 56.15 & 17.09 & 2.80 & 2.25 & 1.28 & 3.61 & 0.83 & n.d. & 5.39 & 0.32 & 220 & 88 & 94 & 49 & 100 & 320 & 21 & 18 & 27 & 160 \\
\hline $416-9-2,12$ & 47.30 & 12.00 & 11.87 & 1.93 & 1.13 & 2.67 & 0.73 & n.d. & 4.93 & 0.50 & 400 & 76 & 71 & 39 & 81 & 480 & 14 & 20 & 21 & 120 \\
\hline $416-10-1,61$ & 49.85 & 18.93 & 2.69 & 2.57 & 1.31 & 3.88 & 0.79 & n.d. & 7.86 & 0.47 & 520 & 87 & 97 & 52 & 110 & 370 & 27 & 26 & 31 & 160 \\
\hline $416-11-3,4$ & 49.35 & 21.77 & 4.51 & 2.94 & 1.25 & 3.60 & 0.73 & n.d. & 6.2 & - & 290 & 160 & 53 & 92 & 210 & 400 & 26 & 19 & 56 & 140 \\
\hline $416-11-3,9$ & 61.95 & 16.34 & 1.78 & 2.24 & 1.68 & 2.90 & 0.68 & n.d. & 5.1 & - & 260 & 150 & 37 & 68 & 160 & 280 & 18 & 17 & 50 & 95 \\
\hline $416-12-1,108$ & 27.75 & 7.04 & 27.93 & 1.76 & 0.89 & 1.64 & 0.30 & n.d. & 4.10 & 0.29 & 810 & 64 & 43 & 34 & 50 & 810 & 14 & 26 & 16 & 47 \\
\hline $416-12-1,147$ & 51.80 & 19.65 & 2.20 & 2.60 & 1.31 & 3.95 & 0.85 & n.d. & 6.75 & 0.58 & 300 & 100 & 95 & 65 & 110 & 380 & 33 & 32 & 28 & 160 \\
\hline $416-13-2,67$ & 41.65 & 18.15 & 8.65 & 2.39 & 1.06 & 3.52 & 0.73 & n.d. & 6.8 & 0.34 & 510 & 88 & 110 & 46 & 110 & 500 & 19 & 17 & 22 & 160 \\
\hline $416-14-1,105$ & 44.05 & 17.11 & 8.05 & 2.69 & 1.06 & 3.45 & 0.83 & n.d. & 7.15 & 0.57 & 510 & 110 & 100 & 56 & 110 & 460 & 22 & 22 & 21 & 168 \\
\hline $416-16-1,25$ & 46.30 & 19.27 & 6.30 & 2.59 & 1.08 & 3.84 & 0.79 & n.d. & 6.1 & 0.20 & 300 & 85 & 110 & 46 & 100 & 460 & 22 & 13 & 18 & 180 \\
\hline $416-18-1,137$ & 35.85 & 11.47 & 18.20 & 2.06 & 0.93 & 2.60 & 0.43 & n.d. & 5.1 & 0.35 & 400 & 110 & 72 & 42 & 74 & 600 & 12 & 9 & 18 & 95 \\
\hline $416-19-2,82$ & 52.80 & 19.99 & 0.46 & 3.15 & 1.30 & 4.52 & 0.81 & n.d. & 7.22 & 0.40 & 320 & 89 & 98 & 60 & 110 & 320 & 31 & 0 & 29 & 160 \\
\hline $416-19-2,101$ & 27.60 & 8.16 & 24.85 & 2.14 & 0.91 & 2.02 & 0.33 & $\mathrm{n}$. & 5.76 & 0.51 & 720 & 66 & 51 & 33 & 53 & 680 & 13 & 0 & 20 & 84 \\
\hline $416-20-1,99$ & 47.65 & 16.90 & 6.23 & 2.65 & 1.26 & 3.31 & 0.72 & n.d. & 6.46 & 0.32 & 390 & 100 & 87 & 47 & 100 & 360 & 23 & 22 & 15 & 140 \\
\hline $416-21-1,10$ & 23.55 & 7.00 & 29.89 & 1.86 & 0.71 & 1.58 & 0.28 & n.d. & 3.7 & 0.35 & 730 & 59 & 42 & 30 & 45 & 770 & 14 & 4 & 18 & 89 \\
\hline $416-23-2,16$ & 29.90 & 10.65 & 21.70 & 2.03 & 0.94 & 2.23 & 0.36 & n.d. & 5.3 & 2.45 & 600 & 98 & 69 & 37 & 67 & 660 & 16 & 35 & 21 & 120 \\
\hline $416-2$ & 51.90 & 18.93 & 1.86 & 2.88 & 1.40 & 3.81 & 0.79 & $\mathrm{n}$. & 7.5 & 0.32 & 450 & 115 & 90 & 57 & 100 & 320 & 28 & 26 & 28 & 160 \\
\hline 1,140 & 28.10 & 9.56 & 26.25 & 2.07 & 0.70 & 1.80 & 0.41 & $\mathrm{n}$. & 3.5 & - & 770 & 130 & 26 & 32 & 95 & 720 & 7 & 31 & 44 & 84 \\
\hline 147 & 48.15 & 20.86 & 2.31 & 3.27 & 1.21 & 3.90 & 0.73 & $\mathrm{n}$. & 8. & - & 420 & 190 & 47 & 170 & 200 & 300 & 33 & 24 & 52 & 120 \\
\hline $416-29$ & 26.30 & 7.73 & 28.00 & 2.12 & 0.71 & 1.60 & 0.21 & $\mathrm{n}$. & 4.3 & 0.32 & 1050 & 58 & 44 & 41 & 47 & 730 & 11 & 34 & 15 & 95 \\
\hline $416-30-5,60$ & 50.35 & 17.00 & 5.71 & 2.91 & 1.36 & 3.69 & 0.73 & n. & 6.72 & 0.22 & 420 & 110 & 81 & 44 & 91 & 410 & 16 & 10 & 14 & 130 \\
\hline $416-36-1,16$ & 56.60 & 13.75 & 6.30 & 2.51 & 1.38 & 2.74 & 0.69 & $\mathrm{n}$. & 5.6 & 0.06 & 410 & 72 & 64 & 32 & 75 & 340 & 12 & 6 & 9 & 110 \\
\hline $416-36-1,129$ & 42.70 & 18.88 & 9.27 & 2.74 & 1.00 & 3.10 & 0.66 & n. & 6. & 0.19 & 420 & 120 & 49 & 77 & 170 & 460 & 22 & 11 & 68 & 120 \\
\hline $416-36-2,8$ & 57.10 & 12.02 & 8.09 & 2.25 & 1.47 & 2.39 & 0.60 & & 4.79 & 0.19 & 480 & 120 & 54 & 35 & 72 & 370 & 18 & 19 & 14 & 95 \\
\hline $416-36-3,28$ & 53.00 & 20.36 & 2.55 & 3.07 & 1.23 & 3.20 & 0.78 & & 6.26 & 0.36 & 320 & 140 & 48 & 95 & 190 & 340 & 23 & 18 & 60 & 130 \\
\hline $416-37-2,77$ & 54.55 & 17.69 & 2.60 & 2.74 & 1.18 & 3.78 & 0.83 & & 6.7 & - & 320 & 89 & 82 & 53 & 102 & 320 & 26 & 25 & 27 & 150 \\
\hline $416-37-3,10$ & 30.40 & 6.59 & 25.24 & 3.20 & 0.64 & 1.57 & 0.30 & n.d. & 4.62 & 0.13 & 720 & 58 & 39 & 26 & 26 & 650 & 10 & 31 & 19 & 79 \\
\hline $416-37-4,7$ & 48.45 & 22.26 & 4.06 & 2.76 & 0.93 & 3.36 & 0.76 & n.d. & 7.01 & 0.11 & 310 & 130 & 61 & 77 & 210 & 370 & 23 & 14 & 63 & 140 \\
\hline $416-38-1,11$ & 48.05 & 15.75 & 10.40 & 2.49 & 1.19 & 3.09 & 0.6 & $n$ & 5. & 0.11 & 370 & 120 & 36 & 41 & 150 & 430 & 15 & 21 & 41 & 95 \\
\hline $416-38-1,44$ & 51.50 & 13.89 & 4.34 & 2.88 & 1.13 & 3.76 & 0.8 & $n$ & 6. & 26 & 310 & 85 & 82 & 51 & 100 & 380 & 23 & 46 & 32 & 140 \\
\hline $416-38-2,18$ & 33.75 & 14.93 & 18.20 & 240 & 0.78 & 2.91 & 0. & $n$ & 5. & 0.19 & 580 & 150 & 36 & 47 & 130 & 550 & 16 & 12 & 63 & 95 \\
\hline $416-40-6,4$ & 55.65 & 14.10 & 3.05 & 2.8 & 1.31 & 3.67 & 0. & & 5. & 0.31 & 330 & 88 & 80 & 51 & 110 & 370 & 32 & 29 & 33 & 150 \\
\hline $416-40-6,17$ & 43.35 & 18.64 & 10.46 & 278 & 0.94 & 3.16 & 0. & & 5. & 0.85 & 400 & 150 & 48 & 47 & 160 & 350 & 16 & 17 & 52 & 120 \\
\hline 416 & 52.40 & 19.94 & 12 & 3.36 & 1.25 & 3.66 & 0.74 & & 7.26 & 0.14 & 300 & 180 & 39 & 81 & 200 & 370 & 25 & 17 & 74 & 120 \\
\hline $416-42-2,125$ & 48.95 & 23.60 & 3.05 & 2.86 & 1.09 & 3.78 & 0.6 & & 7.5 & 0.17 & 310 & 140 & 62 & 87 & 210 & 440 & 26 & 8 & 77 & 140 \\
\hline $416-43-1,46$ & 51.60 & 17.02 & 4.66 & 2.70 & 1.01 & 3.67 & 0.7 & & 7.72 & 0.13 & 390 & 97 & 96 & 51 & 84 & 440 & 26 & 15 & 37 & 150 \\
\hline $416-43-2,51$ & 51.30 & 14.99 & 8.26 & 2.74 & 1.33 & 2.68 & 0.6 & n. & 5.22 & 0.11 & 430 & 140 & 29 & 55 & 140 & 350 & 11 & 22 & 59 & 79 \\
\hline $416-43-4,56$ & 48.85 & 20.30 & 4.55 & 3.19 & 1.13 & 3.80 & 0.73 & & 7.62 & 0.11 & 320 & 130 & 42 & 74 & 180 & 400 & 20 & 13 & 61 & 130 \\
\hline $416-43-4,80$ & 40.80 & 15.81 & 13.05 & 3.11 & 1.33 & 3.06 & 0.5 & & 5.69 & 0.31 & 560 & 190 & 32 & 69 & 160 & 550 & 29 & 47 & 71 & 120 \\
\hline $416-44-1,11$ & 50.30 & 14.75 & 9.31 & 2.49 & 1.35 & 3.23 & 0.6 & & 5.40 & 0.03 & 340 & 100 & 28 & 40 & 150 & 390 & 10 & 17 & & 79 \\
\hline $416-44-2,1$ & 53.60 & 21.59 & 1.22 & 3.36 & 1.31 & 3.98 & 0.8 & n.d. & 5.90 & 0.27 & 280 & 170 & 45 & 150 & 220 & 320 & 33 & 31 & 55 & 140 \\
\hline $416-44-2,7$ & 54.35 & 18.41 & 1.75 & 3.17 & 1.26 & 4.16 & 0.83 & $\mathrm{n}$. & 6.2 & 0.01 & 300 & 95 & 86 & 68 & 97 & 370 & 35 & 18 & 36 & 150 \\
\hline $416-44, \mathrm{CC}, 12$ & 24.70 & 10.50 & 28.35 & 2.61 & 0.66 & 2.28 & 0.3 & & 4.2 & 0.13 & 600 & 130 & 21 & 49 & 100 & 460 & 23 & 14 & 55 & 79 \\
\hline $416-46-2,8$ & 58.00 & 15.69 & 3.96 & 2.48 & 1.50 & 2.95 & 0.7 & & 4. & 0.1 & 340 & 110 & 69 & 41 & 74 & 350 & 22 & 16 & 23 & 100 \\
\hline $416-46-2,10$ & 55.05 & 16.95 & 3.85 & 2.71 & 1.40 & 3.56 & 0.7 & & 5. & 0.17 & 330 & 135 & 89 & 47 & 92 & 360 & 27 & 24 & 33 & 140 \\
\hline $416-46-4,100$ & 48.60 & 17.70 & 4.83 & 3.32 & 1.06 & 3.67 & 0. & & 7. & 0 & 380 & 110 & 76 & 58 & 9 & 400 & 27 & 25 & 32 & 120 \\
\hline $416-46-4,104$ & 60.55 & 15.16 & 2.98 & 2.48 & 1.58 & 2.95 & 0. & & 3. & 0.16 & 310 & 98 & 63 & 38 & 86 & 310 & 13 & 140 & 27 & 95 \\
\hline $416-46-4,110$ & 53.85 & 19.58 & 2.17 & 3.35 & 1.28 & 4.01 & 0.7 & & 5. & 0.19 & 310 & 97 & 88 & 54 & 110 & 390 & 24 & 4 & 29 & 160 \\
\hline $416-48-1,84$ & 42.75 & 15.93 & 10.47 & 3.40 & 1.23 & 3.42 & 0.7 & & 5. & 0.17 & 470 & 130 & 78 & 53 & 77 & 440 & 26 & 30 & 26 & 120 \\
\hline $416-48-3,9$ & 52.35 & 18.29 & 2.91 & 3.11 & 1.20 & 4.11 & 0.7 & & 7. & 0.19 & 330 & 95 & 88 & 62 & 92 & 360 & 32 & 31 & 41 & 140 \\
\hline $416-48-3,85$ & 50.50 & 19.94 & 2.24 & 3.19 & 1.18 & 3.58 & 0. & & 5. & 0 . & 360 & 130 & 93 & 51 & 120 & 370 & 29 & 32 & 27 & 130 \\
\hline $416-48-3,101$ & 53.40 & 19.71 & 2.38 & 2.3 & 1. & 3. & 0. & & 6. & & 370 & 130 & 110 & 47 & 94 & 380 & 22 & 50 & 29 & 130 \\
\hline $416-49-1,6$ & 48.30 & 14.87 & 9. & 2. & & 2. & & & & & 4 & 170 & 7 & 3 & 6 & 510 & 19 & 34 & 46 & 89 \\
\hline $416-49-2,33$ & 43.60 & 9.3 & 17. & 2. & 1. & 2. & & & & & 460 & 9 & 4 & 2 & 4 & 580 & 16 & 15 & 27 & 55 \\
\hline $416-49-2,40$ & 54.40 & 18.2 & 2. & 3. & & 4. & 0. & & & & 370 & 10 & 7 & 5 & 6 & 370 & 23 & 14 & 39 & 150 \\
\hline $416-49-2,109$ & 40.45 & 14. & 13. & 2 . & & & & & & 5 & 460 & 12 & 6 & 4 & 5 & 610 & 19 & 9 & 30 & 89 \\
\hline $416-50-1,1$ & 51.25 & 18.11 & 2.63 & 3. & & 3. & & & & 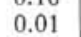 & 330 & 30 & 9 & 7 & 9 & 340 & 29 & 18 & 41 & 120 \\
\hline $416-50-1$ & 21.95 & 7.14 & 31.68 & 1.9 & 0.61 & 1. & 0.2 & n.d. & & 14 & 580 & 15 & 3 & t & 3 & 610 & 13 & 16 & 47 & 53 \\
\hline $416-50-2$ & 48.45 & 11.39 & 13.76 & 1.9 & 0.86 & 2. & 0.54 & n.d. & 2.86 & .19 & 340 & 16 & 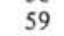 & 2 & 6 & 430 & 9 & 44 & 37 & 58 \\
\hline $416-53-2,3$ & 15.75 & 5.43 & 35.00 & 2.2 & 1.11 & 1.39 & 0.18 & n.d. & 2.14 & 0.14 & 810 & 6 & 2 & 29 & 3 & 840 & 14 & 9 & 43 & 45 \\
\hline $416-53-2,13$ & 52.35 & 17.47 & 4.59 & 3.05 & 1.20 & 4.14 & 0.77 & n.d. & 6.12 & 0.15 & 380 & 85 & 81 & 56 & 103 & 350 & 26 & 14 & 31 & 150 \\
\hline $416-53-2,32$ & 60.10 & 13.92 & 3.40 & 3.15 & 1.77 & 3.77 & 0.64 & n.d. & 4.05 & 0.15 & 360 & 160 & 57 & 34 & 61 & 300 & 13 & 2 & 25 & 63 \\
\hline $416-53-2,142$ & 50.00 & 18.23 & 3.78 & 3.48 & 1.25 & 3.95 & 0.72 & n.d. & 7.15 & 0.18 & 390 & 150 & 77 & 59 & 79 & 350 & 23 & 6 & 39 & 105 \\
\hline $416-57-1,23$ & 50.80 & 19.29 & 2.28 & 3.56 & 1.21 & 4.14 & 0.73 & n.d. & 7.36 & 0.16 & 370 & 150 & 82 & 49 & 100 & 350 & 24 & 32 & 44 & 110 \\
\hline $416-57-1,99$ & 53.45 & 18.39 & 2.66 & 3.37 & 1.18 & 4.28 & 0.79 & n.d. & 6.69 & 0.31 & 370 & 100 & 86 & 53 & 110 & 370 & 26 & 31 & 35 & 150 \\
\hline
\end{tabular}

aOrganic carbon. 


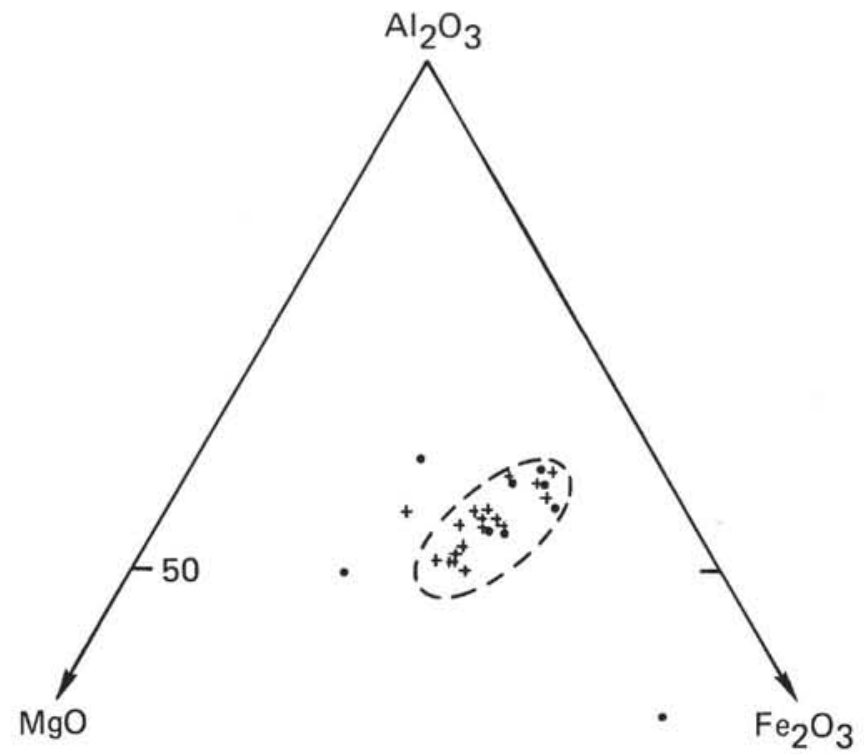

$$
\begin{aligned}
& \text { + = Units I, II, III (Miocene-Pleistocene) } \\
& \text { - = Units IV, V, VI (Albian-Paleocene) }
\end{aligned}
$$

Figure 1. $\mathrm{Al}_{2} \mathrm{O}_{3}-\mathrm{F}_{2} \mathrm{O}_{3}-\mathrm{MgO}$ diagram, Site 415 .

discussed below. It appears to involve enrichment in potassic minerals of the pre-Albian sediments of Site 416 (those minerals being distinctly marked by lithium).

A more distinct comparison between the two sites exists between the samples from the upper part of Hole 416 (Albian-Eocene). All variation domains of the chemical elements overlap (cf. $\mathrm{K}_{2} \mathrm{O}$ and $\mathrm{Li}$ ) and the concentrations of the major components $\left(\mathrm{SiO}_{2}, \mathrm{Al}_{2} \mathrm{O}_{3}\right.$, $\mathrm{CaO}, \mathrm{Fe}_{2} \mathrm{O}_{3}$ ) are the same or very similar to each other. The identity of $\mathrm{Al}_{2} \mathrm{O}_{3}$ and $\mathrm{Fe}_{2} \mathrm{O}_{3}$ confirms a probable analogous clay source. Here the carbonate variations are essentially balanced by changes in the supply of detrital quartz. The similarity is equally obvious in the trace elements (cf. Mn, $\mathrm{Zn}, \mathrm{Ni}, \mathrm{Cr}, \mathrm{Sr}, \mathrm{Co}$ ). Copper, however, at Site 415 and lead at the top of Site 416 are randomly distributed.

We can now confirm that the post-Albian sediments of Sites 415 and 416 are very similar, and that the preAlbian deposits of Site 416 contain more aluminopotassic silicates (which confirms the clay-mineralogy studies of Chamley [this volume]). A greater supply of metalliferous trace elements $(\mathrm{Ti}, \mathrm{Mn}, \mathrm{Li}, \mathrm{Ni})$ was received at Site 416 than at Site 415 .

\section{Factor Analysis}

A mathematical treatment characteristic of factor analysis in the $R$ mode has been applied to the data from the two sites. The data from the two sites are considered separately in order to rapidly determine whether groups of elements (comparable or not) play a particular role in the whole set of geochemical variables.
TABLE 4

\begin{tabular}{|c|c|c|c|c|c|c|c|c|}
\hline $\begin{array}{c}\text { Sample } \\
\text { (interval in } \mathrm{cm} \text { ) }\end{array}$ & $\frac{\mathrm{SiO}_{2}}{\mathrm{Al}_{2} \mathrm{O}_{3}}$ & $\frac{\mathrm{Al}_{2} \mathrm{O}_{3}}{\mathrm{Fe}_{2} \mathrm{O}_{3}}$ & $\frac{\mathrm{MgO}}{\mathrm{K}_{2} \mathrm{O}}$ & $\frac{\mathrm{Al}_{2} \mathrm{O}_{3}}{\mathrm{~K}_{2} \mathrm{O}}$ & $\frac{\mathrm{Na}_{2} \mathrm{O}}{\mathrm{K}_{2} \mathrm{O}}$ & $\frac{\mathrm{Fe}_{2} \mathrm{O}_{3}}{\mathrm{~K}_{2} \mathrm{O}}$ & $\frac{\mathrm{Fe}_{2} \mathrm{O}_{3}}{\mathrm{MgO}}$ & $\frac{\mathrm{Sr} 10^{3}}{\mathrm{CaO}}$ \\
\hline $416-1-1,33$ & 3.3 & 2.3 & 1.1 & 4.5 & 0.92 & 1.9 & 1.80 & 3.3 \\
\hline $416-1-1,44$ & 3.4 & 2.3 & 1.1 & 3.2 & 0.72 & 1.4 & 1.3 & 3.4 \\
\hline $416-1-1,99$ & 5.1 & 2.0 & 1.9 & 3.1 & 0.53 & 2.1 & 0.8 & 3.4 \\
\hline $416-1-1,117$ & 3.9 & 2.2 & 1.3 & 3.8 & 0.67 & 1.7 & 1.4 & 3.4 \\
\hline $416-1-2,33$ & 4.6 & 2.3 & 1.4 & 3.9 & 0.73 & 1.7 & 1.2 & 3.5 \\
\hline $416-2-1,31$ & 4.4 & 2.2 & 1.4 & 3.5 & 0.47 & 1.6 & 1.2 & 3.3 \\
\hline $416-2-3,64$ & 4.8 & 2.2 & 1.2 & 3.4 & 0.56 & 1.6 & 1.4 & 3.4 \\
\hline $416-3-3.39$ & 4.0 & 2.6 & 1.0 & 3.8 & 0.43 & 1.5 & 1.5 & 2.1 \\
\hline $416-3-3,47$ & 4.5 & 2.1 & 1.3 & 3.3 & 0.45 & 1.4 & 1.2 & 3.5 \\
\hline $416-6-3,3$ & 4.6 & 2.9 & 0.5 & 4.0 & 0.40 & 1.7 & 2.7 & 114.3 \\
\hline $416-7-2,122$ & 3.3 & 3.2 & 0.6 & 4.7 & 0.35 & 1.5 & 2.40 & 11.4 \\
\hline $416-9-2,12$ & 3.9 & 2,4 & 0.7 & 4.5 & 0.42 & 1.9 & 2.6 & 4.0 \\
\hline $416-10-1,61$ & 2.6 & 2.4 & 0.7 & 4.9 & 0.34 & 2.0 & 3.1 & 13.8 \\
\hline $416-11-3,4$ & 2.3 & 3.5 & 0.8 & 6.1 & 0.35 & 1.7 & 2.1 & 8.8 \\
\hline $416-11-3,9$ & 3.8 & 3.2 & 0.8 & 5.6 & 0.58 & 1.8 & 2.3 & 15.7 \\
\hline $416-12-1,108$ & 3.9 & 1.7 & 1.1 & 4.3 & 0.54 & 2.5 & 2.3 & 2.9 \\
\hline $416-12-1,147$ & 2.6 & 2.9 & 0.7 & 5.0 & 0.33 & 1.7 & 2.6 & 17.3 \\
\hline $416-13-2,67$ & 2.3 & 2.6 & 0.7 & 5.2 & 0.30 & 2.0 & 2.9 & 5.8 \\
\hline $416-14-1,105$ & 2.6 & 2.4 & 0.8 & 5.0 & 0.31 & 2.1 & 2.7 & 5.7 \\
\hline $416-16-1,25$ & 2.4 & 3.1 & 0.7 & 5.0 & 0.28 & 1.6 & 2.4 & 7.3 \\
\hline $416-18-1,137$ & 3.1 & 2.2 & 0.8 & 4.4 & 0.36 & 2.0 & 2.6 & 3.3 \\
\hline $416-19-2,82$ & 2.6 & 2.8 & 0.7 & 4.4 & 0.29 & 1.6 & 2.3 & 69.6 \\
\hline $416-19-2,101$ & 3.4 & 1.4 & 1.1 & 4.0 & 0.45 & 2.9 & 2.7 & 2.7 \\
\hline $416-20-1,99$ & 2.8 & 2.6 & 0.8 & 5.1 & 0.38 & 2.0 & 2.4 & 5.8 \\
\hline $416-21-1,10$ & 3.4 & 1.9 & 1.2 & 4.4 & 0.45 & 2.4 & 2.0 & 2.6 \\
\hline $416-23-2,16$ & 2.8 & 2.0 & 0.9 & 4.8 & 0.42 & 2.4 & 2.6 & 3.0 \\
\hline $416-25-1,97$ & 2.7 & 2.5 & 0.8 & 5.0 & 0.37 & 2,0 & 2.6 & 17.2 \\
\hline $416-25-1,140$ & 2.9 & 2.7 & 1.2 & 1.3 & 0.4 & 2.0 & 1.7 & 2.7 \\
\hline $416-25-1,147$ & 2.3 & 2.4 & 0.8 & 5.4 & 0.31 & 2.0 & 2.6 & 13.2 \\
\hline $416-29-3,23$ & 3.4 & 1.8 & 1.3 & 4.8 & 0.44 & 2.2 & 2.0 & 2.6 \\
\hline $416-33-5,60$ & 3.0 & 2.5 & 0.8 & 4.6 & 0.37 & 2.7 & 2.3 & 7.2 \\
\hline $416-36-1,16$ & 4.1 & 2.4 & 0.9 & 5.0 & 0.5 & 1.8 & 2.3 & 5.4 \\
\hline $416-36-1,129$ & 2.3 & 2.7 & 0.9 & 6.1 & 0.32 & 2.1 & 2.5 & 5.0 \\
\hline $416-36-2,8$ & 4.8 & 2.5 & 0.9 & 5.0 & 0.62 & 2.0 & 2.1 & 4.6 \\
\hline $416-36-3,28$ & 2.6 & 3.3 & 1.0 & 6.4 & 0.38 & 2.0 & 2.0 & 13.3 \\
\hline $416-37-2,77$ & 3.1 & 2.6 & 0.7 & 4.7 & 0.31 & 1.8 & 2.5 & 12.3 \\
\hline $416-37-3,10$ & 4.6 & 1.4 & 2.0 & 4.2 & 0.41 & 2.9 & 1.4 & 2.6 \\
\hline $416-37-4,7$ & 2.2 & 3.2 & 0.8 & 6.6 & 0.28 & 2.1 & 2.5 & 9.1 \\
\hline $416-38-1,11$ & 3.1 & 3.0 & 0.8 & 5.1 & 0.39 & 1.7 & 2.1 & 4.1 \\
\hline $416-38-1,44$ & 3.7 & 2.1 & 0.8 & 3.7 & 0.30 & 1.8 & 2.3 & 8.8 \\
\hline $416-38-2,18$ & 2.3 & 2.6 & 0.8 & 5.1 & 0.27 & 2.0 & 2.4 & 3.0 \\
\hline $416-40-6,4$ & 4.0 & 2.4 & 0.8 & 3.8 & 0.36 & 1.6 & 2.0 & 12.1 \\
\hline $416-40-6,17$ & 2.3 & 3.5 & 0.9 & 5.9 & 0.30 & 1.7 & 1.9 & 3.4 \\
\hline $416-40-6,22$ & 2.6 & 2.8 & 0.9 & 5.4 & 0.34 & 2.0 & 2.2 & 11.9 \\
\hline $416-42-2,125$ & 2.1 & 3.1 & 0.8 & 6.2 & 0.29 & 2.0 & 2.6 & 1.3 \\
\hline $416-43-1,46$ & 3.0 & 2.2 & 0.7 & 4.6 & 0.28 & 2.9 & 2.9 & 9.4 \\
\hline $416-43-2,51$ & 3.4 & 2.9 & 1.0 & 5.6 & 0.5 & 2.0 & 1.9 & 4.2 \\
\hline $416-43-4,56$ & 2.4 & 2.7 & 0.8 & 5.3 & 0.30 & 2.0 & 2.4 & 8.9 \\
\hline $416-43-4,80$ & 2.6 & 2.8 & 1.0 & 5.2 & 0.43 & 1.9 & 1.8 & 18.0 \\
\hline $416-44-1,11$ & 3.4 & 2.7 & 0.8 & 4.6 & 0.42 & 1.7 & 2.2 & 4.2 \\
\hline $416-44-2,1$ & 2.5 & 3.7 & 0.8 & 5,4 & 0.33 & 1.5 & 1.8 & 26.2 \\
\hline $416-44-2,7$ & 3.0 & 3.0 & 0.8 & 4.4 & 0.30 & 1.5 & 2.0 & 21.1 \\
\hline $416-44, \mathrm{CC}, 12$ & 2.4 & 2.5 & 1,11 & 4.6 & 0.29 & 1.9 & 1.6 & 1.6 \\
\hline $416-46-2,8$ & 3.7 & 3.8 & 0.8 & 5.3 & 0.51 & 1.4 & 1.7 & 8.8 \\
\hline $416-46-2,10$ & 3.3 & 3.2 & 0.8 & 4.8 & 0.39 & 1.5 & 2.0 & 9.4 \\
\hline $416-46-4,100$ & 2.8 & 2.3 & 0.9 & 4.8 & 0.29 & 2.1 & 2.3 & 8.3 \\
\hline $416-46-4,104$ & 4.0 & 4.0 & 0.8 & 5.1 & 0.54 & 1.3 & 1.5 & 10.4 \\
\hline $416-46-4,110$ & 2.8 & 3.5 & 0.8 & 4.9 & 0.32 & 1.4 & 1.7 & 18.0 \\
\hline $416-48-1,84$ & 2.7 & 2.8 & 1.0 & 4.7 & 0.36 & 1.7 & 1.7 & 4.2 \\
\hline $416-48-3,9$ & 2.9 & 2.5 & 0.8 & 4.4 & 0.29 & 1.8 & 2.3 & 12.4 \\
\hline $416-48-3,85$ & 2.5 & 3.6 & 0.9 & 5.6 & 0.33 & 1.6 & 1.7 & 16.5 \\
\hline $416-48-3,101$ & 2.7 & 3.1 & 0.7 & 5,8 & 0.32 & 1.3 & 2.7 & 16.0 \\
\hline $416-49-1.6$ & 3.2 & 3.1 & 0.9 & 5.7 & 0.45 & 1.9 & 2.1 & 5.2 \\
\hline $416-49-2,33$ & 4,7 & 2.9 & 0.9 & 3.8 & 0.34 & 1.3 & 1.5 & 3.3 \\
\hline $416-49-2,40$ & 3.0 & 2.5 & 0.8 & 4.4 & 0.37 & 1.8 & 2.2 & 18.5 \\
\hline $416-49-2,109$ & 2.7 & 3.0 & 0.9 & 4.8 & 0.35 & 1.6 & 1.8 & 4.5 \\
\hline $416-50-1.1$ & 2.8 & 2.3 & 0.8 & 4.6 & 0.4 & 2.0 & 2.5 & 12.9 \\
\hline $416-50-1,14$ & 3.1 & 2.3 & 1.2 & 4.5 & 0.39 & 1.9 & 1.6 & 1.9 \\
\hline $416-50-2,2$ & 4.3 & 4.0 & 0.9 & 5.0 & 0,38 & 1.3 & 1.4 & 3.1 \\
\hline $416-53-2,3$ & 2.9 & 2.5 & 1.6 & 3.9 & 0.80 & 1.5 & 0.9 & 2.4 \\
\hline $416-53-2,13$ & 3.0 & 2.9 & 0.7 & 4.2 & 0.29 & 1.5 & 2.0 & 7.6 \\
\hline $416-53-2,32$ & 4.3 & 3.4 & 1.1 & 5.0 & 0.64 & 1.5 & 1.3 & 8,8 \\
\hline $416-53-2,142$ & 2.7 & 2.6 & 0.9 & 4.6 & 0.32 & 1.8 & 2.1 & 9.3 \\
\hline $416-57-1,23$ & 2.6 & 2.6 & 0.9 & 4.7 & 0.29 & 1.8 & 2.1 & 15.4 \\
\hline $416-57-1,99$ & 2.9 & 2.8 & 0.8 & 4.3 & 0.28 & 1.6 & 2.0 & 13.9 \\
\hline
\end{tabular}

Geochemical Ratios, Site 416

We have limited our study to the search for factors on orthogonal axes using an iterative method on the correlations matrix (factor analysis in principal components). The calculation was carried out for five factors. 


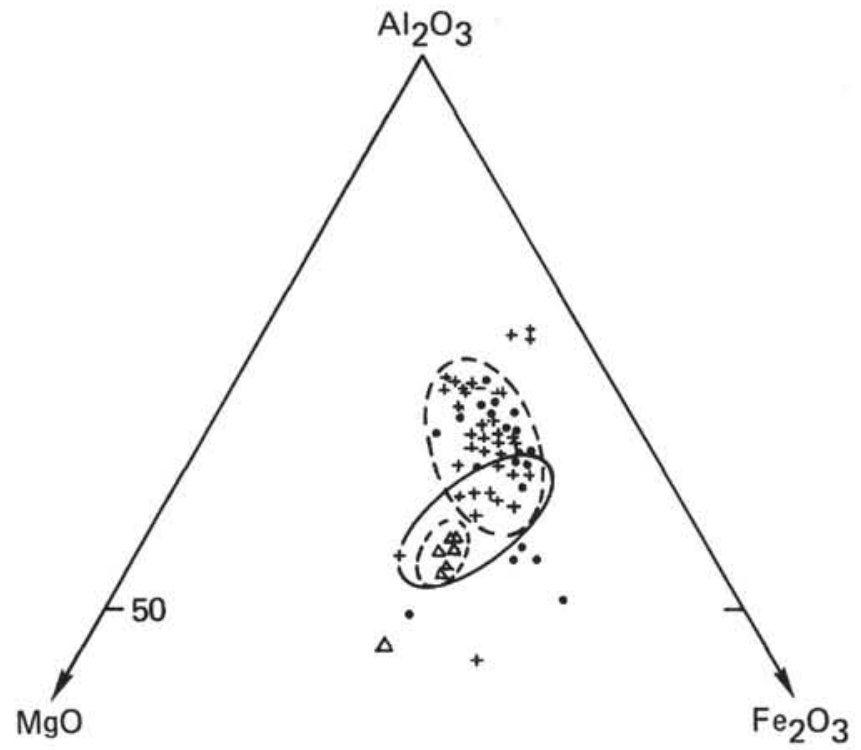

$$
\begin{aligned}
\Delta & =\text { Units I, II (Eocene-Pleistocene) } \\
\bullet & =\text { Unit VI (Valanginian-Barremian) } \\
+ & =\text { Unit VII (Tithonian-Valanginian) } \\
& =\text { Site } 415 \text { data }
\end{aligned}
$$

\begin{tabular}{|c|c|c|c|c|c|c|}
\hline & \multicolumn{2}{|c|}{ Site 415} & \multicolumn{2}{|c|}{$\begin{array}{c}\text { Site } 416 \\
\text { (all samples) }\end{array}$} & \multicolumn{2}{|c|}{$\begin{array}{c}\text { Site } 416 \\
\text { (Albian- } \\
\text { Pleistocene) }\end{array}$} \\
\hline & $\overline{\mathrm{x}}$ & $\sigma$ & $\overline{\mathrm{X}}$ & $\sigma$ & $\overline{\mathrm{X}}$ & $\sigma$ \\
\hline $\mathrm{SiO}_{2}(\%)$ & 30.9 & 14.2 & 45.3 & 11.4 & 35.2 & 11.7 \\
\hline $\mathrm{Al}_{2} \mathrm{O}_{3}$ & 8.2 & 4.1 & 14.9 & 4.8 & 8.3 & 2.6 \\
\hline $\mathrm{CaO}$ & 24.6 & 12.4 & 10.5 & 10 & 20.5 & 10.6 \\
\hline $\mathrm{MgO}$ & 2.2 & 0.8 & 2.7 & 0.6 & 3.0 & 1.0 \\
\hline $\mathrm{Na}_{2} \mathrm{O}$ & 1.3 & 0.4 & 1.2 & 0.3 & 1.3 & 0.2 \\
\hline $\mathrm{K}_{2} \mathrm{O}$ & 1.3 & 0.5 & 3.1 & 0.8 & 2.3 & 0.7 \\
\hline $\mathrm{TiO}_{2}$ & 0.44 & 0.23 & 0.62 & 0.20 & 0.35 & 0.18 \\
\hline $\mathrm{Fe}_{2} \mathrm{O}_{3}$ & 3.6 & 1.8 & 5.6 & 1.5 & 3.7 & 1.0 \\
\hline $\mathrm{Mn}(\mathrm{ppm})$ & 320 & 170 & 420 & 150 & 340 & 40 \\
\hline $\mathrm{Zn}$ & 75 & 27 & 113 & 40 & 84 & 18 \\
\hline $\mathrm{Li}$ & 35 & 9 & 64 & 24 & 53 & 16 \\
\hline $\mathrm{Ni}$ & 34 & 9 & 51 & 25 & 29 & 7 \\
\hline $\mathrm{Cr}$ & 76 & 45 & 102 & 47 & 73 & 31 \\
\hline $\mathrm{Sr}$ & 850 & 380 & 470 & 190 & 700 & 350 \\
\hline Co & 13 & 4 & 20 & 7 & 10 & 4 \\
\hline $\mathrm{Cu}$ & 31 & 56 & 25 & 17 & 27 & 13 \\
\hline $\mathrm{Pb}$ & 24 & 17 & 41 & 38 & 72 & 100 \\
\hline V & 100 & 47 & 113 & 35 & 79 & 31 \\
\hline
\end{tabular}

Figure 2. $\mathrm{Al}_{2} \mathrm{O}_{3}-\mathrm{Fe}_{2} \mathrm{O}_{3}-\mathrm{MgO}$ diagram, Site 416 .

TABLE 5

Mean Chemical Compositions, Leg 50 Sites

Note: $\overline{\mathrm{X}}=$ mean concentration; $\sigma=$ root mean square deviation.

\section{Site 415 (Figure 3)}

Owing to the small number of samples and to the lack of dispersion of the data, five factors account for nearly 89 per cent of the global variance.

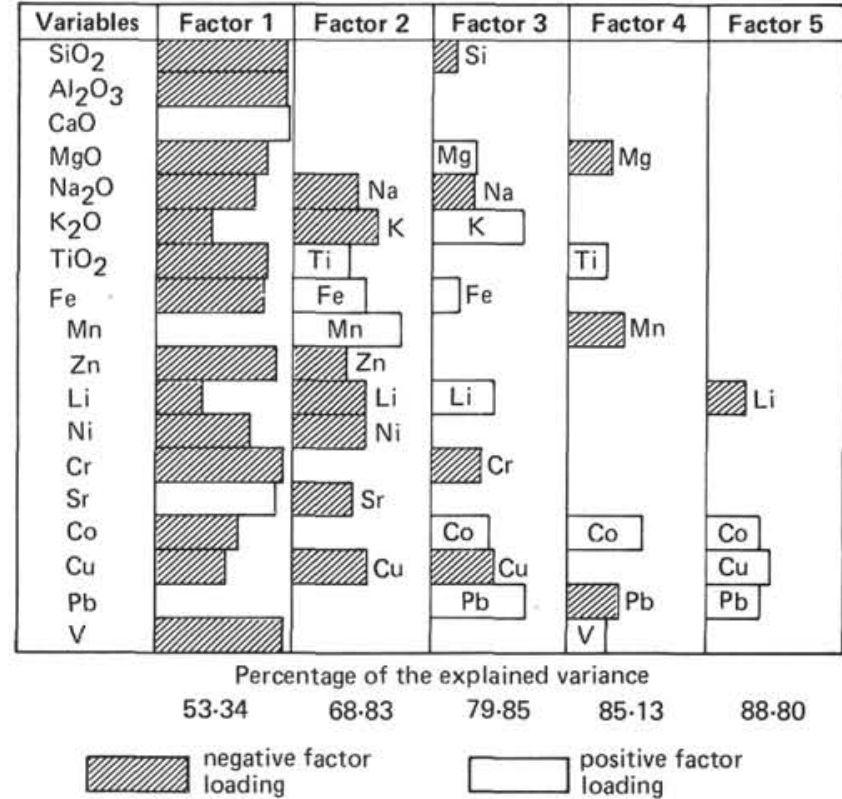

Figure 3. R mode factor analysis, Site 415 analyses.

Except for $\mathrm{K}_{2} \mathrm{O}$ among the major elements, and $\mathrm{Mn}$, $\mathrm{Li}$, and $\mathrm{Pb}$ among the trace elements, all the variables occur heavily weighted in the first factor, which represents 53 per cent of the global variance. This factor can be defined as the negative correlation between the strontian carbonate phase and the aluminosilicate phases. The relationship is made evident by the signs affecting each variable.

If we arrange the variables of the first factor in order of weights for the major elements $\left(\mathrm{Al}_{2} \mathrm{O}_{3}, \mathrm{SiO}_{2}, \mathrm{MgO}\right.$, $\left.\mathrm{Fe}, \mathrm{Na}_{2} \mathrm{O}, \ldots\right)$ and then for the trace elements $(\mathrm{Cr}, \mathrm{V}$, $\mathrm{Zn}, \mathrm{Ti}, \mathrm{Ni}, \ldots$ ), we see in the silicates the dominant ferromagnesian character of the smectite-fibrous clay mixture defining the clay base of most of the samples (at least below the Miocene). Furthermore, we note that most of the trace elements are preferentially tied to the clay minerals $(\mathrm{Cr}, \mathrm{V}, \mathrm{Zn}, \mathrm{Ti}, \mathrm{Ni}, \mathrm{Co})$, whereas $\mathrm{Mn}$ and $\mathrm{Pb}$ seem to be independent.

The second factor is marked by a negative correlation between the groups $\mathrm{Mn}-\mathrm{Fe}$ on one hand and $\mathrm{K}_{2} \mathrm{O}, \mathrm{Li}$, $\mathrm{Ni}, \mathrm{Cu}$ on the other. Most of these elements are poorly represented in the first factor. We interpret this as an opposition between the supply of potassic clay minerals marked by lithium and nickel, and metalliferous precipitations in an oxidizing environment ( $\mathrm{Fe}, \mathrm{Mn})$. This second factor might be characteristic of the post-Eocene period because we detected illitic contributions from the Miocene upwards. Moreover, the occurrence of most manganese is related with carbonate to the top of that series, whereas the iron and manganese most frequently co-exist in the oxide form. (Goethite is reported by Chamley [this volume] in Sample 415B-2-1, $44 \mathrm{~cm}$.)

The third factor is defined only by a potassic phase apparently marked by lithium, lead, and cobalt. Cobalt occurs ubiquitously and seems to be distributed among the alumino-ferrous and potassic silicates. 
Factors 4 and 5 are not very explicit because each are defined by one single element of sufficent weight: cobalt (factor 4) and copper (factor 5). Each element behaves a particular way.

\section{Site 416 (Figure 4)}

Five factors explain only 78 per cent of the global variance, which confirms the heterogeneity of the samples collected at that site.

As for Site 415, the first factor is characterized by the negative correlation between the silicate and carbonate phases, but the composition of those phases is distinct at the two sites. In arranging the variables of the first factor in order of decreasing weights of the same sign, we obtain $\mathrm{K}_{2} \mathrm{O}, \mathrm{Al}_{2} \mathrm{O}_{3}, \mathrm{SiO}_{2}, \mathrm{Fe}, \mathrm{MgO}, \mathrm{Na}_{2} \mathrm{O}, \ldots$ for the major elements and $\mathrm{Ti}, \mathrm{V}, \mathrm{Co}, \mathrm{Cr}, \mathrm{Ni}, \mathrm{Li}, \ldots$ for the trace elements. The dominant clay base is thus clearly alumino-potassic and ferruginous instead of magnesian, whereas lithium and manganese play a large role in the general covariance. The carbonate phase is marked by strontium and to a lesser degree by manganese. The presence of manganese at that level is a second difference from the Site 415 sequence.

The second factor, defined by trace elements, shows the negative correlation between the $(\mathrm{Ni}, \mathrm{Cr}, \mathrm{Pb})$ group and lithium; those elements mark different clay-mineral phases. The presence of lead, however, is difficult to explain inasmuch as lead is practically independent at the level of the first factor.

The third factor is marked by a negative correlation between sodium and the vanadium-manganese couple, but the interpretation of that correlation is still unexplained.

In the fourth factor, the lead-strontium linkage results because part of the lead is tied to the carbonates.

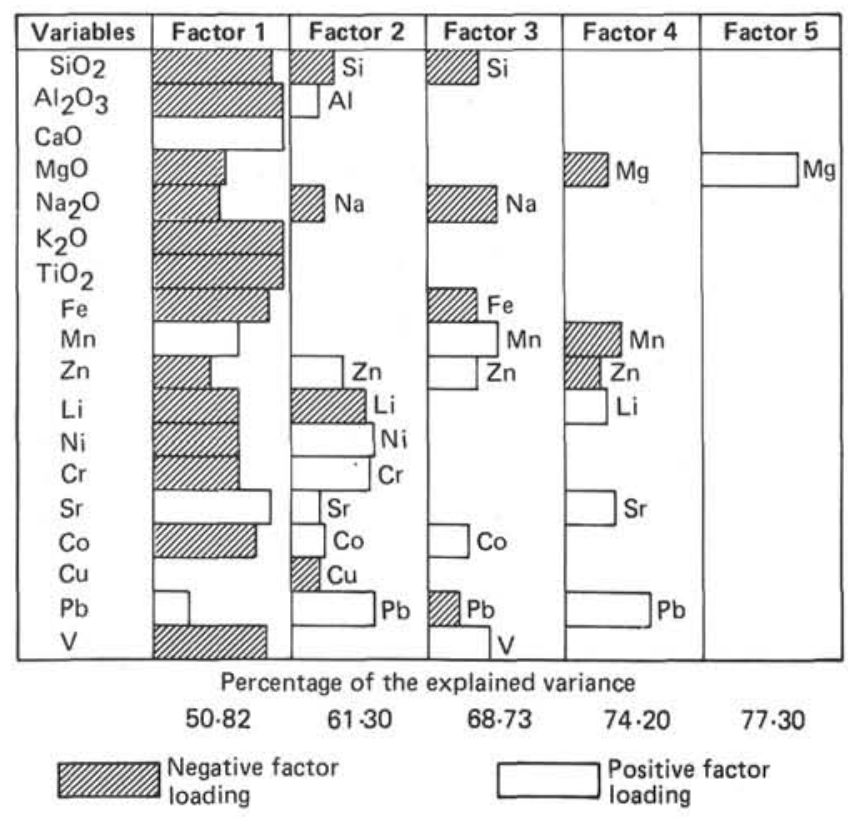

Figure 4. R mode factor analysis, Site 416 analyses.
This relationship could account for the sign observed for lead in the first factor.

Lastly, the fifth factor is, as at Site 415 , defined by the fluctuation of copper, which is a particularly independent element in the various sites that have been studied.

\section{Total Correlations}

In this study, we deal with properties being either characteristic of each site or common to both of them. Only the correlation coefficients that were significant at a probability threshold of 0.01 (Fisher test) have been retained; the range of variation is too great from 0.02 onward. Most of the relationships elicited above are also found in this aspect of the study.

\section{Site 415 (Table 6)}

The major silicate phase is defined by the grouping of highly covariant oxides $\mathrm{Al}_{2} \mathrm{O}_{3}\left(\mathrm{SiO}_{2}, \mathrm{Fe}_{2} \mathrm{O}_{3}, \mathrm{MgO}\right)$ reflecting the clear dominance of ferro-magnesian silicates (smectite) which constitute the larger part of the clay mineral assemblage of the pre-Miocene samples.

A close examination of the demonstrated interrelationships reveals the existence of a sodic silicate phase marked by zinc, and one that contains the small amounts of copper seen in the core: $\mathrm{SiO}_{2}\left(\mathrm{Na}_{2} \mathrm{O}, \mathrm{Zn}, \mathrm{Cu}\right)$. The particular covariance of $\mathrm{Na}_{2} \mathrm{O}$ and its intense linkage with silica were previously found in fibrous-clay levels at Site 398 (Debrabant et al., unpublished manuscript).

An apparently independent potassic phase indicates completely different origins for illite and smectite, which is confirmed by metalliferous markers. Lithium marks the potassic phase only, whereas titanium, chromium, and vanadium mark the ferromagnesian phase. Nickel is ubiquitous; cobalt, weakly covariant, tends to mark alumino-ferrous levels.

Chromium and silica $\left(R^{1}=0.97\right)$ are particularly strongly related and also occur in the post-Albian sediments of Site 416 (Figure 5).

Also the $\mathrm{Al}_{2} \mathrm{O}_{3}-\mathrm{TiO}_{2}$ couple from Miocene sediments upward form an almost linear relationship; the data are more scattered in older sediments. Finally, manganese and lead seem to be totally independent. In the preMiocene sediments (Figure 6) manganese is mainly bound to calcium carbonates, but in Miocene sediments and upward it seems to occur only in the oxidized state. This could result from diagenesis caused by a more intense degree of oxidation in the shallower sediments.

Lastly, the $\mathrm{CaO}-\mathrm{Sr}$ association is in opposition to the silicate phase. However, the association of the two elements of this classical couple seems to be discontinuous (Figure 7). The data points seem to align along two separate straight lines, and show a break somewhere between the Paleocene and Miocene samples. For identical amounts of lime, strontium concentrations in-

\footnotetext{
${ }^{1} R=$ correlation coefficient.
} 
TABLE 6

Probable Correlations (limit 0.01 - Fisher's Test), Site 415

\begin{tabular}{|c|c|c|c|c|c|c|c|c|c|c|c|c|c|c|c|c|c|}
\hline & $\mathrm{SiO}_{2}$ & $\mathrm{Al}_{2} \mathrm{O}_{3}$ & $\mathrm{CaO}$ & $\mathrm{MgO}$ & $\mathrm{Na}_{2} \mathrm{O}$ & $\mathrm{K}_{2} \mathrm{O}$ & $\mathrm{TiO}_{2}$ & $\mathrm{Fe}_{2} \mathrm{O}_{3}$ & $\mathrm{Mn}$ & $\mathrm{Zn}$ & $\mathrm{Li}$ & $\mathrm{Ni}$ & $\mathrm{Cr}_{\mathrm{I}}$ & $\mathrm{Sr}$ & $\mathrm{Co}$ & $\mathrm{Cu}$ & $\mathrm{Pb}$ \\
\hline $\mathrm{Al}_{2} \mathrm{O}_{3}$ & 0.87 & & & & & & & & & & & & & & & & \\
\hline $\mathrm{CaO}$ & -0.98 & -0.94 & & & & & & & & & & & & & & & \\
\hline $\mathrm{MgO}$ & 0.73 & 0.80 & -0.80 & & & & & & & & & & & & & & \\
\hline $\mathrm{Na}_{2} \mathrm{O}$ & 0.70 & 0.58 & -0.67 & & & & & & & & & & & & & & \\
\hline \multicolumn{18}{|l|}{$\mathrm{K}_{2} \mathrm{O}$} \\
\hline $\mathrm{TiO}_{2}$ & 0.73 & 0.94 & -0.82 & 0.65 & & & & & & & & & & & & & \\
\hline $\mathrm{Fe}_{2} \mathrm{O}_{3}$ & 0.68 & 0.85 & -0.73 & 0.66 & & & 0.87 & & & & & & & & & & \\
\hline \multicolumn{18}{|l|}{$\mathrm{Mn}^{2}$} \\
\hline $\mathrm{Zn}$ & 0.88 & 0.74 & -0.84 & & 0.81 & & & & & & & & & & & & \\
\hline $\mathrm{Li}$ & & & & & & 0.78 & & & & & & & & & & & \\
\hline $\mathrm{Ni}$ & 0.64 & & -0.63 & 0.61 & 0.76 & 0.60 & & & & 0.75 & & & & & & & \\
\hline $\mathrm{Cr}$ & 0.97 & 0.81 & -0.94 & 0.67 & 0.74 & & 0.64 & 0.61 & & 0.85 & & 0.63 & & & & & \\
\hline $\mathrm{Sr}$ & -0.87 & -0.85 & 0.89 & -0.69 & & & -0.81 & -0.86 & & -0.66 & & & -0.82 & & & & \\
\hline Co & & 0.62 & & & & & 0.58 & 0.63 & & & & & & & & & \\
\hline $\mathrm{Cu}$ & 0.55 & & & & 0.71 & & & & & 0.73 & & & 0.66 & & & & \\
\hline \multicolumn{18}{|l|}{$\mathrm{Pb}$} \\
\hline V & 0.82 & 0.93 & -0.87 & 0.65 & 0.61 & & 0.86 & 0.82 & & 0.71 & & & 0.79 & -0.89 & 0.60 & & \\
\hline
\end{tabular}

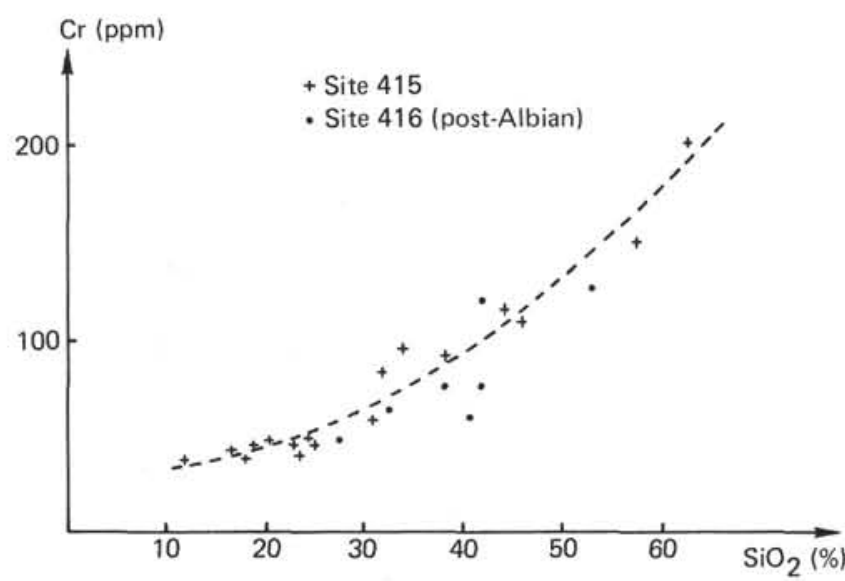

Figure 5. Variation of Cr content as a function of $\mathrm{SiO}_{2}$ content, Sites 415 and 416.

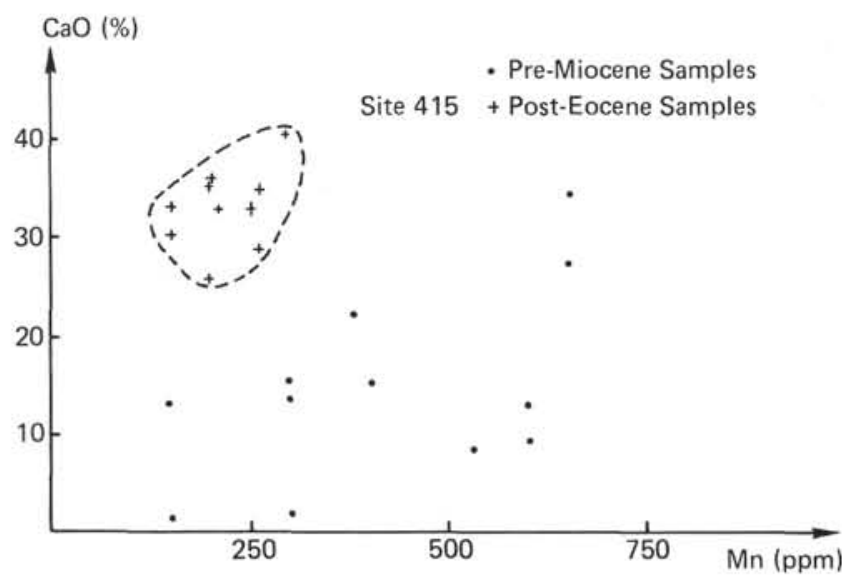

Figure 6. Variation of $\mathrm{CaO}$ content as a function of $\mathrm{Mn}$ content, Site 415 . crease markedly from the Miocene sediments upwards. Here again the carbonate could be of diagenetic origin or it could reflect the more pelagic character of the environment.

\section{Site 416 (Table 7)}

The study of total correlations for this site shows that the most covariant set is an alumino-potassic and ferriferous phase $\mathrm{Al}_{2} \mathrm{O}_{3}\left(\mathrm{~K}_{2} \mathrm{O}, \mathrm{Fe}_{2} \mathrm{O}_{3}, \mathrm{TiO}_{2}\right)$. Magnesium seems to intervene to a much lesser degree in the whole core. However, the relationships within the trace elements are much the same as at Site 415.

Though it plays a large part in the general covariance, lithium shows its closest linkage with the potassic phase (represented throughout the sampling). Titanium is closely bound to the whole set of alumino-ferriferous and potassic clay minerals. However, its linkage with aluminum is not a simple one. $\mathrm{TiO}_{2}$ increases regularly together with aluminum up to the level of 14 per cent of $\mathrm{Al}_{2} \mathrm{O}_{3}$. Beyond that percentage, the $\mathrm{TiO}_{2}$ content becomes stable between 0.65 per cent and 0.85 per cent (Figure 8). This phenomenon cannot be explained easily, because extra quantities of aluminum can originate from a clay mineral not readily susceptible to titanium replacements.

Nickel, chromium, and cobalt seem to be ubiquitous, whereas vanadium behaves like titanium. Last, copper and lead rank in secondary position, whereas copper is totally independent.

The carbonate phase, marked not only by strontium but also by manganese is, of course, in opposition to the silicate phase. This relation is far from being a persistent one (Figure 9); it disappears from the Eocene and above. Strontium behaves very much the same way (Figure 7). The graphic representation of $\mathrm{Sr}$ versus $\mathrm{CaO}$ produces, as for Site 415 , two separate straight lines when pre- and post-Paleocene samples are separated. 


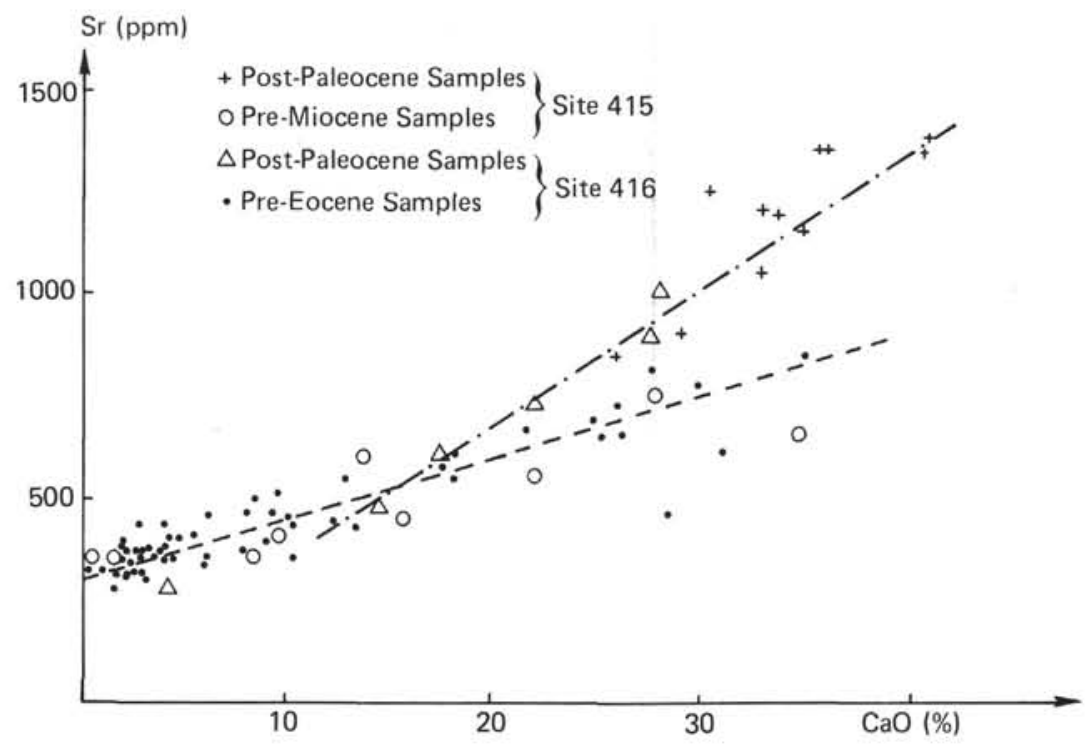

Figure 7. Variation of $\mathrm{Sr}$ content as a function of $\mathrm{CaO}$ content, Sites 415 and 416.

\section{CONCLUSIONS}

We cannot easily compare the two sites ( 415 and 416 ) of Leg 50 because of the stratigraphic difference of the two series concerned. For example, the ferro-potassic character of Site 416 is largely contributed by preAlbian sediments which are absent from Site 415. Site 415 has a marked ferromagnesian character. Certain criteria, however, which may indicate that Site 415 material is more characteristic of a more-oceanic environment than that of Site 416, are worth noting. Furthermore, an environmental change which seems to correspond to more open sea conditions occurred simultaneously at both sites after the Paleocene. Our data are also comparable with the results obtained at the more northerly sites (398 and 400). At those sites, the major alumino-ferriferous phase is marked by the same grouping of trace elements ( $\mathrm{Ti}, \mathrm{V}, \mathrm{Cr}$ ), whereas lithium tends to characterize the more potassic minerals (which was previously noted in Leg 47B).

Fibrous clay minerals (Site 415) are accompanied (as at Site 398) by a stronger covariance of sodium and magnesium. Below the Tertiary sediments the carbonate phase, which is inversely related to the silicate phase, is marked not only by strontium but also by manganese. This tendency was also noted in Site 398 samples.

Metalliferous precipitations occur in Albian to Eocene sediments from the deeper site of the Bay of Biscay. The stratigraphic limits of that phenomenon corresponded with intervals rich in fibrous clay minerals and

TABLE 7

Probable Correlations (limit 0.01 - Fisher's Test), Site 416

\begin{tabular}{|c|c|c|c|c|c|c|c|c|c|c|c|c|c|c|c|c|c|}
\hline & $\mathrm{SiO}_{2}$ & $\mathrm{Al}_{2} \mathrm{O}_{3}$ & $\mathrm{CaO}$ & $\mathrm{MgO}$ & $\mathrm{Na}_{2} \mathrm{O}$ & $\mathrm{K}_{2} \mathrm{O}$ & $\mathrm{TiO}_{2}$ & $\mathrm{Fe}_{2} \mathrm{O}_{3}$ & $\mathrm{Mn}$ & $\mathrm{Zn}$ & $\mathrm{Li}$ & $\mathrm{Ni}$ & $\mathrm{Cr}$ & $\mathrm{Sr}$ & Co & $\mathrm{Cu}$ & $\mathrm{Pb}$ \\
\hline $\mathrm{Al}_{2} \mathrm{O}_{3}$ & 0.72 & & & & & & & & & & & & & & & & \\
\hline $\mathrm{CaO}$ & -0.95 & -0.87 & & & & & & & & & & & & & & & \\
\hline $\mathrm{MgO}$ & 0.37 & 0.40 & -0.48 & & & & & & & & & & & & & & \\
\hline $\mathrm{Na}_{2} \mathrm{O}$ & 0.64 & 0.30 & -0.57 & 0.34 & & & & & & & & & & & & & \\
\hline $\mathrm{K}_{2} \mathrm{O}$ & 0.79 & 0.88 & -0.91 & 0.56 & 0.45 & & & & & & & & & & & & \\
\hline $\mathrm{TiO}_{2}$ & 0.85 & 0.87 & -0.92 & 0.33 & 0.45 & 0.88 & & & & & & & & & & & \\
\hline $\mathrm{Fe}_{2} \mathrm{O}_{3}$ & 0.55 & 0.83 & -0.74 & 0.43 & & 0.82 & 0.74 & & & & & & & & & & \\
\hline $\mathrm{Mn}^{2}$ & -0.68 & -0.51 & 0.65 & -0.31 & -0.59 & -0.60 & -0.57 & & & & & & & & & & \\
\hline $\mathrm{Zn}$ & & 0.46 & -0.35 & 0.30 & & 0.32 & & 0.33 & & & & & & & & & \\
\hline $\mathrm{Li}$ & 0.51 & 0.50 & -0.61 & & & 0.66 & 0.62 & 0.55 & -0.35 & & & & & & & & \\
\hline $\mathrm{Ni}$ & 0.35 & 0.69 & -0.50 & 0.34 & & 0.54 & 0.50 & 0.62 & & 0.51 & & & & & & & \\
\hline $\mathrm{Cr}$ & 0.44 & 0.73 & -0.56 & 0.33 & & 0.53 & 0.51 & 0.56 & -0.41 & 0.48 & & 0.78 & & & & & \\
\hline $\mathrm{Sr}$ & -0.88 & -0.74 & 0.89 & -0.50 & -0.44 & -0.78 & -0.79 & -0.63 & 0.47 & -0.40 & -0.45 & -0.42 & -0.52 & & & & \\
\hline Co & 0.44 & 0.74 & -0.61 & 0.34 & & 0.73 & 0.70 & 0.71 & -0.32 & 0.34 & 0.49 & 0.68 & -0.44 & -0.46 & & & \\
\hline \multicolumn{18}{|l|}{$\mathrm{Cu}$} \\
\hline $\mathrm{Pb}$ & -0.34 & & 0.32 & & & & & & & & -0.39 & & & 0.52 & & & \\
\hline V & 0.55 & 0.78 & -0.71 & 0.31 & & 0.79 & 0.79 & 0.79 & -0.35 & & 0.72 & 0.47 & 0.46 & -0.57 & 0.73 & & \\
\hline
\end{tabular}




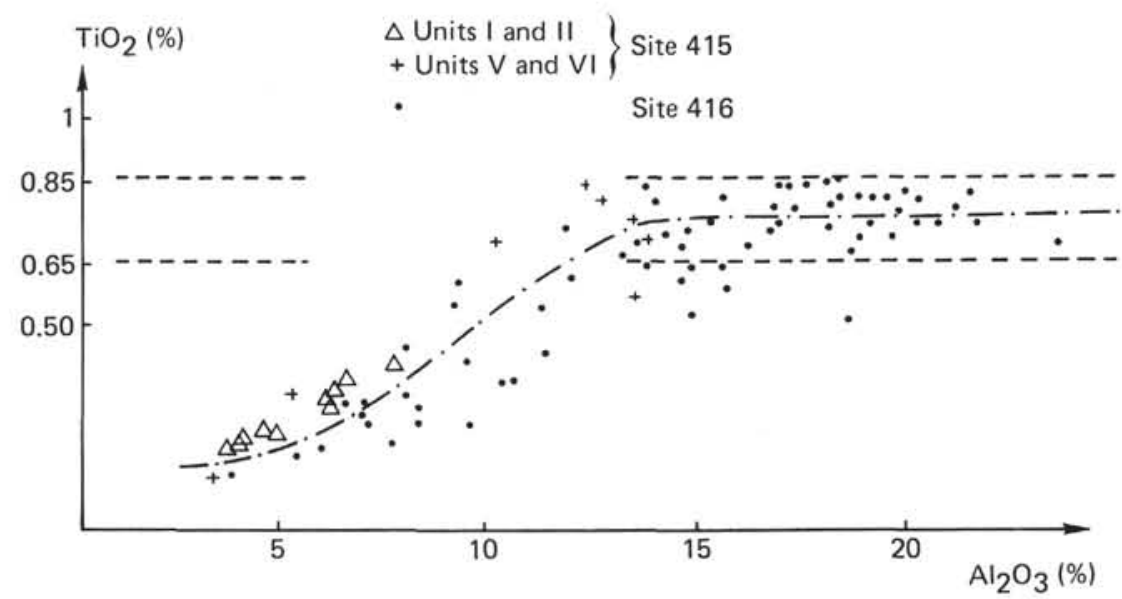

Figure 8. Variation of $\mathrm{TiO}_{2}$ content as a function of $\mathrm{Al}_{2} \mathrm{O}_{3}$ content, Sites 415 and 416.

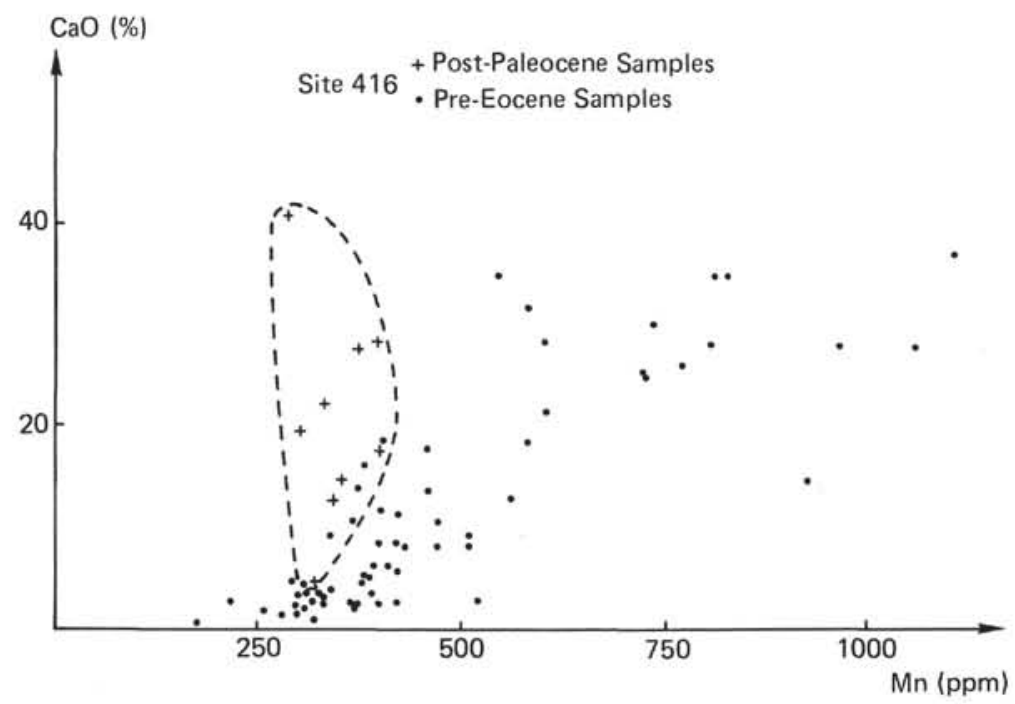

Figure 9. Variation of $\mathrm{CaO}$ content as a function of $\mathrm{Mn}$ content, Site 416.

may have been caused by deep currents. Nothing similar was noted in the Leg 50 material, not even that from Site $\mathbf{4 1 5}$ which is rich in attapulgite. We thus formulate two hypotheses. Depths at Leg 50 sites were either approximately the same as at Site 402 (Leg 48), or the metalliferous carrier originated from the north and encountered the fibrous-clay carrier in the area of the Bay of Biscay, thus favoring the precipitation of transition elements.

\section{ACKNOWLEDGMENTS}

We are grateful to the U. S. National Science Foundation and to the shipboard team of Leg 50 for allowing us to conduct the investigations. We received financial support for the study from CNRS (France) through Grants ATP-IPOD. The geochemical study of carbon was realized thanks to the technical assistance of $\mathrm{R}$. Jouglet and the mathematical treatment was worked by $\mathbf{M}$. Meunier. We gratefully acknowledge W. R. Riedel and $\mathrm{R}$. Létolle for reviewing the manuscript.

\section{REFERENCES}

Chamley, H., Debrabant, P., Foulon, J., Giroud D'Argoud, G., Latouche, C., Maillet, N., Maillot, H., and Sommer, F., 1979. Mineralogy and geochemistry of Cretaceous and Cenozoic Atlantic sediments of the Iberian peninsula (Site 398, DSDP Leg 47B). In Ryan W., Sibuet, J.-C., et al., Initial Reports of the Deep Sea Drilling Project, v. 47, Part 2: Washington (U.S. Government Printing Office).

Debrabant, P., Chamley, H., Foulon, J., and Maillot, H., 1979. Mineralogy and geochemistry of Upper Cretaceous and Cenozoic sediments from North Biscay Bay and Rockall Plateau (Eastern North Atlantic), DSDP Leg 48. In Montadert, L., Roberts, D.G., et al., Initial Reports of the Deep Sea Drilling Project, v. 48: Washington (U.S. Government Printing Office), p. 703-726.

Deer, W.A., Howie, R.A., and Zussman, J., 1963. Rock forming minerals, Sheet silicates: (Longmans), v.3. 